\title{
Programas matinales televisivos: un análisis cuantitativo de las entrevistas a políticos en TVE y Antena 3*
}

\section{Itziar Reguero-Sanz ${ }^{1}$ Virginia Martín-Jiménez ${ }^{2}$}

Recibido: 02/04/2019

Aprobado por pares: 29/08/2019
Enviado a pares: 03/04/2019

Aceptado: 06/09/2019

DOI: $10.5294 /$ pacla.2020.23.1.5

\section{Para citar este artículo/to reference this article/para citar este artigo}

Reguero-Sanz, I. y Martín-Jiménez, V. (2020). Programas matinales televisivos: un análisis cuantitativo de las entrevistas a políticos en TVE y Antena 3. Palabra Clave, 23(1), e2315. https://doi.org/10.5294/pacla.2020.23.1.5

\section{Resumen}

Este artículo analiza los programas matinales de entrevistas y debates de Televisión Española (TVE) y Antena 3, emitidos entre 1997 y 2006, en una doble vertiente. En primer lugar, se lleva a cabo un examen cualitativo de la trayectoria de Los desayunos, El primer café, La respuesta y Ruedo ibérico y de la situación empresarial y política de las cadenas que los emitían. A continuación, a través de un muestreo, se realiza un estudio cuantitativo de los políticos que fueron entrevistados en estos programas para analizar la presencia de los diferentes partidos e ideologías en estos espacios y trazar una comparativa entre la cadena pública y la privada. Los resultados revelan que ambos canales tuvieron una agenda muy similar, optaron por la diversidad ideológica desde un punto de vista cuantitativo y por los partidos con

Este artículo se ha realizado dentro de los siguientes proyectos de investigación: "Historia de la programación y de los programas de televisión en España (cadenas de ámbito estatal): de la desregulación al apagón analógico, 1990-2010" (CSO2015-66260-C4-1-P), financiado por el Ministerio de Economía y Competitividad (España); y "Perfiles del centro político (1976-1986): proyectos y realizaciones" (HAR2016-75600-C2-P), financiado por el Ministerio de Economía y Competitividad (España) (AI, FEDER, UE).

1 https://orcid.org/0000-0002-3274-1861. Universidad de Valladolid, España. itziar.reguero@uva.es

2 https://orcid.org/0000-0002-7099-1060. Universidad de Valladolid, España. virginia.martin@uva.es 
mayor peso parlamentario. En conclusión, se puede determinar, gracias a esta investigación, que los matinales examinados de la cadena pública y la privada fueron espacios televisivos muy similares, tanto en su estructura y funcionamiento interno como en el perfil de los invitados relacionados con la política que fueron entrevistados.

\section{Palabras clave (Fuente: tesauro de la Unesco)}

Programa de televisión; entrevista; partidos políticos; político; España. 


\section{The television morning programs: A quantitative analysis of the interviews with politicians on TVE and Antena 3}

\section{Abstract}

The present investigation analyzes the TVE and Antena 3 morning talk shows, broadcasted between 1997 and 2006, in two aspects. On the one hand, a qualitative examination of the trajectory of Los desayunos, El primer café, La respuesta and Ruedo ibérico, and the business and political situation of the broadcasting chains is carried out. On the other hand, through a sampling, a quantitative study of the politicians interviewed in these programs is realized, in order to analyze the presence of the different parties and ideologies in these TV shows and draw a comparison between the public and private chain. The results reveal that both channels had a very similar agenda, opting for ideological diversity from a quantitative point of view and for the parties with greater parliamentary weight. In conclusion, it can be determined, thanks to this investigation, that the exam of the morning programs of the public and private channels were very similar television spaces, both in their structure and in their internal functioning, as in the profile of the guests related to the policy that were interviewed.

\section{Keywords (Source: Unesco Thesaurus)}

Television programmes; interviews; political parties; politicians; Spain.

* This article was made within these research projects: "Historia de la programación y de los programas de televisión en España (cadenas de ámbito estatal): de la desregulación al apagón analógico, 1990-2010” (CSO2015-66260C4-1-P), financed by the Ministerio de Economía y Competitividad (Spain); and "Perfiles del centro político (1976-1986): proyectos y realizaciones” (HAR2016-75600-C2-P), finianced by the Ministerio de Economía y Competitividad (Spain) (AI, FEDER, UE). 


\section{Programas matinais de televisão: uma análise quantitativa das entrevistas a políticos na TVE e no Antena 3}

\section{Resumo}

Este artigo analisa os programas matinais de entrevistas e debates de Televisión Española (TVE) e Antena 3, emitidos entre 1997 e 2006, em uma dupla vertente. Em primeiro lugar, é realizado um exame qualitativo da trajetória de Los desayunos, El primer café, La respuesta e Ruedo ibérico, e da situação empresarial e política de suas emissoras. A seguir, por meio de uma amostra, é realizado um estudo quantitativo dos políticos que foram entrevistados nesses programas para analisar a presença dos diferentes partidos e ideologias nesses espaços e estabelecer uma comparação entre a emissora pública e a privada. Os resultados evidenciam que ambos os canais tiveram uma agenda muito similar, optaram pela diversidade ideológica do ponto de vista quantitativo e pelos partidos com maior peso parlamentar. Em conclusão, pode-se determinar, graças a esta pesquisa, que os programas analisados da emissora pública e da privada foram espaços televisivos muito semelhantes, tanto em sua estrutura e funcionamento interno quanto no perfil dos convidados entrevistados relacionados com a política.

\section{Palavras-chave (Fonte: tesauro da Unesco)}

Programa de televisão; entrevista; partidos políticos; político; Espanha.

\footnotetext{
* Este artigo foi realizado dentro dos projetos de pesquisa: "Historia de la programación y de los programas de televisión en España (cadenas de ámbito estatal): de la desregulación al apagón analógico, 1990-2010" ("História da programação e dos programas de televisão na Espanha (emissoras do governo): da desregulamentação ao apagão analógico, 1990-2010" - CSO2015-66260-C4-1-P), financiado pelo Ministério de Economia e Competitividade da Espanha, e "Perfiles del centro político (1976-1986): proyectos y realizaciones" ("Perfis do centro político (1976 1986): projetos e realizações" - HAR2016-75600-C2-P), financiado pelo mesmo ministério (AI, FEDER, UE).
} 


\section{La política en los espacios televisivos matinales: planteamiento y justificación de la investigación}

En 1994, Televisión Española (TVE) puso en marcha Los desayunos para ofrecer a la audiencia matinal contenido de actualidad política a través del debate y las entrevistas. El formato de este espacio marcará tendencia y se consolidará en la programación televisiva en la que desde su origen la información y la política habían tenido un peso significativo en diferentes franjas de la parrilla televisiva (Montero y Antona, 2018; Martín, 2018).

La apuesta por Los desayunos tuvo lugar en plena efervescencia mediática tras la irrupción de las cadenas privadas al inicio de la década de 1990, lo que influyó de manera directa en las estrategias de programación desplegadas (Gómez-Escalonilla, 2003). El liderazgo en cuanto a número de espectadores que ejercía la cadena estatal llevó a Antena 3 (líder de audiencia en el ámbito de las cadenas privadas) a tratar de competir a través de la búsqueda de una serie de productos de calidad en consideración a los intereses de la audiencia española por aquel entonces. "No interesaba competir de forma directa con los productos de Telecinco (programas espectáculo entre los que destacaban las Mamachicho como emblema de la emisora durante estos años) sino que se pretendía ofrecer una programación estructurada en tres pilares" que le permitiese hacer frente a la televisión pública (García, 2014a, p. 84).

Uno de esos pilares - y como columna vertebral de la estrategia programática - fueron los programas informativos diarios y semanales en toda su variedad, incluidos los debates de actualidad y las entrevistas (García, 2015). De ahí que en 1996 Antena 3 apostase por competir de manera directa con el formato matinal de actualidad política de TVE poniendo en marcha, en la misma franja horaria que Los desayunos, el programa El primer café que se prolongará en el tiempo en la parrilla, aunque, como veremos, modificando su título (El primer café, La respuesta y Ruedo ibérico).

Estos productos televisivos en los que se centra esta investigación presentan una estructura muy semejante entre ellos, pues contaban tanto con una entrevista a un invitado (preferentemente relacionado con el mundo 
de la política) como con un debate sobre un tema de actualidad. A su vez, y siguiendo con los paralelismos, ambos disfrutaron de altos índices de audiencia en dos cadenas líderes en share (tabla 1).

\section{Tabla 1. Evolución del share televisivo de TVE, La 2 y Antena 3 (1997-2006)}

\begin{tabular}{|c|c|c|c|c|c|c|c|c|c|c|}
\hline & $\mathbf{1 9 9 7}$ & $\mathbf{1 9 9 8}$ & $\mathbf{1 9 9 9}$ & $\mathbf{2 0 0 0}$ & $\mathbf{2 0 0 1}$ & $\mathbf{2 0 0 2}$ & $\mathbf{2 0 0 3}$ & $\mathbf{2 0 0 4}$ & $\mathbf{2 0 0 5}$ & $\mathbf{2 0 0 6}$ \\
\hline TVE & 25,1 & 25,5 & 24,9 & 24,5 & 24,9 & 24,7 & 23,4 & 21,9 & 19,6 & 18,2 \\
\hline La 2 & 8,9 & 8,8 & 8,0 & 7,9 & 7,8 & 7,7 & 7,2 & 6,9 & 5,8 & 4,9 \\
\hline Antena 3 & 22,7 & 22,7 & 22,6 & 21,4 & 20,3 & 20,2 & 19,5 & 20,8 & 21,3 & 19,4 \\
\hline
\end{tabular}

Fuente: TNS Sofres, Sofres audiencia de medios y Kantar Media, citados por García (2014b, pp. 274 y 335).

Como veremos con detalle, todos estos matinales estuvieron muy sujetos a la polémica. Así, cuatro de sus presentadoras (Ana Pastor y María Casado en TVE y Consuelo Álvarez de Toledo e Isabel San Sebastián en Antena 3) tuvieron una controvertida salida de los programas por motivos que afectaban a su libertad a la hora de informar. También se percibe que tanto los periodistas como los invitados que acudían a los espacios oscilaban entre los diferentes programas y cadenas, por lo que se puede advertir una competencia directa entre ellos.

Por otra parte, cabe destacar que en la Comisión de Control Parlamentario de RTVE se abordaron sucesivas veces cuestiones que tenían que ver con la presencia de ideologías políticas en los programas matinales de actualidad. Prueba de ello son, por ejemplo, las sesiones en las que se trataron temas como los criterios de selección de los invitados (Diario de sesiones del Congreso de los Diputados, 1996, p. 1239) o el número de veces en que los políticos del grupo mixto (entre otros) habían participado en las tertulias de TVE (Diario de sesiones del Congreso de los Diputados, 2001, p. 4541).

Entre estos ejemplos encontramos que el programa Los desayunos fue también objeto de debate en dichas sesiones. En concreto, en marzo de 1998, se planteaba en el orden del día el siguiente punto: "Criterio con el que se seleccionan a los invitados de Los desayunos de RTVE” (Diario de sesiones del 
Congreso de los Diputados, 1998, p. 16203). En el diálogo parlamentario de esta sesión, el director general del ente público RTVE respondía a la cuestión planteada explicando que "hay evidentemente una serie de personajes políticos y sociales de enorme peso en sí mismos, que además responden al pluralismo y la pluralidad existente en nuestra sociedad política española”; mientras que en junio de 1997 el Diario de sesiones recoge otra intervención de la Comisión de Control Parlamentario en la que se afirma: "Esta es la imparcialidad. [... ] Le voy a dar un ejemplo. En los famosos desayunos de Radio Nacional de España el PSOE, el 20 por ciento, Izquierda Unida, el 7 por ciento; Gobierno, administración y afines, 47 por ciento" (Diario de sesiones del Congreso de los Diputados, 1997, p. 6949). Vemos, por tanto, que la presencia y el peso de las diferentes agrupaciones políticas fue un tema que preocupaba a los políticos (Humanes y Fernández, 2015). Una preocupación que también estaría presente en el caso de los contenidos de Antena 3, aunque no contasen con la posibilidad que ofrecía la televisión pública de incluir esta cuestión en el debate parlamentario.

A partir de lo expuesto, y en consideración a esa etapa de emergencia de las televisiones privadas en un contexto de cambio político a partir de 1996 y la puesta en marcha de estrategias de programación que permitiesen captar a una audiencia nunca antes tan segmentada, esta investigación busca estudiar los programas matinales de entrevistas y debates de TVE y Antena 3, emitidos entre 1997 y 2006, en una doble vertiente. En primer lugar, se traza una aproximación a la trayectoria de los espacios matinales a los que hemos hecho referencia y a la situación empresarial y política de las cadenas que los emitían, para, posteriormente, llevar a cabo un estudio cuantitativo de los políticos que fueron entrevistados en dichos programas, a fin de analizar la presencia de los diferentes partidos e ideologías en este tipo de formatos y trazar una comparativa entre ambas cadenas en consideración al carácter público y privado de cada una de ellas. ${ }^{3}$

3 Cuando arranca este estudio, gobernaba el PP tras conseguir el poder en 1996. Durante la primera legislatura de la Presidencia de José María Aznar, la dirección general de Radiotelevisión Española (RTVE) la asumió Mónica Ridruejo (1996-1997), quien fue reemplazada por Fernando López-Amor (1997-1998), diputado en el Congreso (PP), quien poco después fue sustituido por Pío Cabanillas (1998-2000) (A. Gómez, 2006). Una vez consiguió mayoría absoluta el Gobierno del PP, Cabanillas se convirtió en ministro portavoz de la Presidencia y llegó a la dirección de RTVE José González Ferrari (2000-2002), a quien le sucedió José Antonio Sánchez Domínguez (2002- 


\section{Objetivos e hipótesis}

El objetivo principal de esta investigación es estudiar los programas matinales de entrevistas y debates que TVE y Antena 3 emitieron durante los diez primeros años de existencia de este formato televisivo tras la ruptura del monopolio: Los desayunos, El primer café, La respuesta y Ruedo ibérico. Primeramente, se hará una aproximación cualitativa a la historia de estos espacios para - como núcleo central del trabajo - llevar a cabo un examen cuantitativo que permita conocer cuál fue el peso de la presencia de políticos en las entrevistas que tuvieron lugar dentro de cada uno de los programas objeto de estudio, qué partidos contaron con minutos en estos programas $y$, por último, qué transmiten estos datos aplicando una perspectiva de género (Vinuesa, Abejón y Sánchez, 2011). Todo ello se hará planteando un doble análisis longitudinal en el tiempo por cadena y, a su vez, un estudio comparado entre los datos que arroja la muestra de TVE y Antena 3.

En atención a estos objetivos se ha partido de las siguientes hipótesis:

1. Tanto la cadena pública como la privada ofrecerán entrevistas a aquellos políticos que tienen más poder de actuación en la esfera parlamentaria y que, por tanto, marcan la agenda de la actualidad política: los representantes del Gobierno y de la oposición (Partido Popular [PP] y Partido Socialista Obrero Español [PSOE], y viceversa, a partir de 2004) y los partidos nacionalistas (con peso político también a lo largo de las legislaturas en las que se desarrollaron las emisiones que se analizan y la casuística diferencial, en cuanto al calendario electoral de las autonomías a las que están vinculados).

2. Antena 3 y TVE cuidarán contar desde un punto cuantitativo con una presencia equilibrada de ideologías políticas que les ayudaría a soportar una imagen de cadenas que dan voz a partidos diferentes al margen de gobiernos o intereses empresariales.

3. La convergencia de los resultados entre ambos canales refleja cómo la actualidad política fija la agenda informativa y marca los conteni- 
dos de estos programas matinales, y genera un producto audiovisual, al menos en apariencia, semejante.

\section{Metodología}

Tras llevar a cabo una aproximación cualitativa a la historia de los espacios televisivos que protagonizan este trabajo, se ha realizado un análisis de contenido cuantitativo para lograr los objetivos propuestos. La muestra de estudio son los programas matinales que se emitieron en TVE (Los desayunos) y en Antena 3 (El primer café, La respuesta y Ruedo ibérico) entre 1997 y 2006, ambos inclusive. Si bien es cierto que se analizan tres programas de la cadena privada frente a uno de la pública, esto no supone un problema metodológico por las siguientes razones:

- Los espacios de Antena 3 tienen las mismas características entre ellos y se encuadran dentro de lo que hemos definido como programas matinales de debate y entrevistas, solo que se modifica el título.

- $\quad$ En el caso de Los desayunos, esta estrategia también se desarrolla, aunque no se perciba de forma tan clara. Para reinventar los matinales, TVE en vez de cambiar el nombre completo (como en Antena 3) solo modifica el final: Los desayunos de Radio 1, Los desayunos de Radio Nacional y Los desayunos de TVE.

- Y la última y más importante razón es que, en todo caso, el objetivo de esta investigación no es tanto analizar cada uno de los programas como un caso específico aislado de la parrilla que ofrece cada cadena, sino estudiar las estrategias que utilizaban tanto TVE como Antena 3 para enfocar sus espacios matinales desde el punto de vista de la ideología política. De ahí que se analice esta doble mirada desde un canal público y otro privado.

En cuanto a la franja temporal analizada, el estudio se centra en los primeros diez años (1997-2006) tras la irrupción de las cadenas privadas durante los cuales TVE y Antena 3 coinciden en la programación matinal con un programa de debates y entrevistas de actualidad política. La muestra se compone de cuatro semanas completas (de lunes a domingo) de cada 
año que están ubicadas en marzo, julio, septiembre y diciembre. Los meses seleccionados buscan representar la programación en diferentes épocas del año: marzo y septiembre con la puesta en marcha de la nueva temporada (primavera y otoño, respectivamente), julio con el comienzo de la parrilla de verano y diciembre con lo emitido en la etapa navideña (García, 2014a). Durante todo el periodo de estudio se han analizado —en consideración a la clasificación de macrogéneros y géneros televisivos de Euromonitor- 225 programas matinales en total, 118 emitidos en TVE y 107 en Antena 3 (anexo 1).

Para extraer los datos de la investigación cualitativa, se han visionado espacios aleatorios a partir del muestreo y se ha recurrido a la prensa de referencia nacional, más concretamente a la sección televisiva. En lo referido a la parte cuantitativa, la información se ha extraído de las parrillas televisivas del diario $A B C$, ya que eran las más completas y pormenorizadas de las publicadas en la prensa escrita.

\section{Una aproximación cualitativa a los programas analizados}

\section{Los desayunos}

El primer matinal de información política en TVE fue Los desayunos de Radio 1. Este espacio comenzó a emitirse simultáneamente en La 2 de TVE y en Radio Nacional de España a partir del 10 de enero de 1994. Dirigido y moderado por Julio César Iglesias, este debate político contaba con la participación de Diego Carcedo, entonces director de la cadena radiofónica estatal, y Antonio San José $(A B C, 1994)$. El programa se emitía de lunes a viernes con una duración de 50 min (9:10-10:00 de la mañana).

En 1996, y ya con Ernesto Sáenz de Buruaga como director de informativos, cambió su nombre por Los desayunos de Radio Nacional. En esta nueva etapa, Alejo García y Javier González Ferrari acompañaron en la mesa de debate a Iglesias. Con el inicio de la siguiente temporada, en septiembre de 1997, se estableció definitivamente su nombre actual: Los desayunos de TVE. 
Luis Mariñas se hizo con las riendas del programa en septiembre de 1999; después de ocho años de trabajo en Telecinco, volvió a la que había sido su casa durante dos décadas. Mariñas permaneció en el programa durante seis temporadas junto con un elenco de colaboradores: Carlos Dávila, Charo Zarzalejos y Fernando Jáuregui, entre otros. En el periodo final de Mariñas (septiembre de 2001-marzo de 2004), este espacio formaba parte del Telediario matinal de TVE. ${ }^{4}$

A partir de septiembre de 2004, Los desayunos volvieron a tener autonomía en la parrilla, coincidiendo con la llegada de Pepa Bueno, quien se comprometió a conducir una tertulia "plural, sosegada y tranquila", y que ofreciese un análisis de la actualidad (El País, 2004). La periodista estaba acompañada ante las cámaras por varios colaboradores, entre ellos, Ignacio Escolar, Anabel Díez, Victoria Prego y Enric Sopena.

Bueno comenzó a presentar el Telediario en horario nocturno, por lo que tuvo que ser sustituida en Los desayunos. Ana Pastor asumió el relevo entre septiembre de 2009 y julio de 2012, junto con Jesús Maraña, Joaquín Estefanía y Nativel Preciado, entre otros profesionales de la información. La salida de Pastor en 2012 fue muy polémica: la propia periodista señaló que su cesión poco tenía que ver con una cuestión laboral: "Me destituyen por hacer periodismo y por una decisión política" [...] "No quieren decir que es un despido, pero es así. No estoy dispuesta a cobrar dinero público por hacer pasillos" (Gómez, 2012).

María Casado sustituyó a Ana Pastor, tras su paso por 59 segundos $(A B C, 2012)$, por el Telediario del fin de semana y por El debate de La 1. La presentadora mantuvo su puesto hasta septiembre 2016. También fue polémica la salida de Casado, ya que ella misma pidió abandonar las mañanas de TVE "harta de presiones". A partir de entonces tomó las riendas Sergio Martín durante dos años (El Confidencial, 2016). Actualmente, el espacio lo dirige Xabier Fortes y se ha añadido una segunda parte: Más de-

4 La entrevista más vista de la última temporada fue la de José Luis Rodríguez Zapatero, que coincidió con el 11-M: 1258000 espectadores. 
sayunos, "una clara apuesta por reforzar la información de actualidad y recuperar el pulso informativo de La 1 en la franja matinal" (TVE, 2018). ${ }^{5}$

\section{El primer café}

Si en la etapa anterior, con el Grupo Zeta al frente (1992-1997), Antena 3 había tratado de atraer a la audiencia a través de programas espectáculo y fichajes millonarios (García, 2014a, p. 82), con la llegada del Grupo Telefónica a la dirección de la cadena (1997-2003), y con José María Mas Millet en la presidencia, en la emisora se advirtió "una preocupación menor por los contenidos televisivos, de los cuales pocos consiguieron más allá de un interés momentáneo por parte de la audiencia, sin lograr consolidarse en la parrilla” (García, 2014b, p. 262), como fue el caso de los programas matinales que protagonizan esta investigación. Sin embargo, posteriormente, a partir de 2003, al adquirir la dirección el Grupo Planeta y la presidencia José Manuel Lara, la estrategia empresarial se orientó principalmente hacia la búsqueda de la rentabilidad (p. 342).

En atención a esa estrategia empresarial de la década de 1990, Antena 3, para competir con TVE, estrenó en octubre de 1996 El primer café. Estaba presentado por Antonio San José, quien había formado parte de la primera etapa de Los desayunos, y por Consuelo Álvarez de Toledo. Su duración estaba en torno a una hora (en ocasiones 55 min y en otras hasta 75) y comenzaba alrededor de las 9:30 de la mañana.

Este espacio constaba de varios bloques: repasaba las noticias del día de la mano de un invitado, interpretaba los titulares de los periódicos, conectaba en directo con las tertulias de radio y abría una línea para la participación de los oyentes. Entre los colaboradores del programa en su primera etapa se encontraban José Oneto, Nativel Preciado y Victoria Prego (estas últimas serían tertulianas posteriormente de Los desayunos).

En enero de 1997, Álvarez de Toledo dimitió por discrepancias con la línea editorial de la cadena. En un comunicado, Antena 3 considera "ino-

5 Para aproximarse a este programa, no hay que olvidar que forma parte de la programación de una televisión pública cuya trayectoria, desde los inicios de su historia, ha estado siempre muy marcada por los diferentes gobiernos estatales (A. Gómez, 2006). De ahí que sea interesante que el lector considere lo que se ha señalado en la nota 2. 
pinada y sorprendente" la dimisión y subraya que "todas las personas que participan en El primer café cuentan con absoluta libertad para manifestar sus opiniones" (El País, 1997). Pero no hubo vuelta atrás. Desde entonces el espacio fue presentado en exclusiva por San José hasta junio de 1998. Coincidiendo con la nueva temporada, tomó el relevo Isabel San Sebastián quien abandonó TVE para fichar por el canal privado (El País, 1998). Los colaboradores más reseñables de este periodo fueron Carlos Herrera y Pedro J. Ramírez.

Debido a este último, tras cuatro años, Antena 3 despediría a Isabel San Sebastián. César Alierta, presidente de Telefónica y principal accionista del canal privado, prohibió la presencia de Pedro J. debido a un conflicto con él. San Sebastián se mostró en contra de esta medida y fue cesada, alegando "decisiones empresariales" $(A B C, 2002)$. La periodista mostró su disconformidad e indignación por este hecho:

Es una decisión que acaté pero sobre la cual expresé mi disconformi-
dad y me han echado. En nuestro mundo no se puede ser indepen-
diente. Ya en el pasado quisieron echar a un compañero tertuliano
pero me negué, y no pasó nada. [...] Me parece una represalia por
mantener mi criterio de independencia sobre una decisión que no me
parece justa ni adecuada. (La Vanguardia, 2002)

Carmen Gurruchaga tomaría las riendas en septiembre 2002, momento en que se incluiría en el espacio "Buenos días, España”. Las cifras del programa cada vez eran más bajas (12,9\%) y, en consecuencia, más alejadas de su principal competidor Los desayunos, que tenían un $24,8 \%$ de share (Gallo, 2003a). El espacio consumó su desaparición en el verano de 2003 y pronto Antena 3 encontró un sustituto para ocupar esta franja de la parrilla.

\section{La respuesta}

El nuevo matinal tenía un corte informativo muy similar a su antecesor. Tanto es así que coincidimos con $A B C$ en que eran el mismo programa con diferente nombre: "El primer café', de Antena 3, pasa a llamarse 'La respuesta" $(A B C, 2003)$. El espacio, dirigido y moderado por Javier González Ferrari, entonces presidente de Onda cero (Gallo, 2003b), combinaba las entrevistas y el análisis de la actualidad política en 45 min (de 9:15 a 10:00). 
A mitad de la primera temporada, y a causa de una enfermedad, Ferrari fue sustituido por Gurruchaga, subdirectora del programa, y desde marzo lo hizo Pedro Piqueras, cuya intención era "remodelar el espacio, que en su última etapa mantiene una escasísima media del $10 \%$ de cuota de pantalla" (El Mundo, 2004) por debajo de sus más directos competidores Los desayunos y La mirada crítica.

No obstante, esta nueva etapa duró apenas dos meses, ya que a Piqueras lo nombraron director de Radio Nacional de España $(A B C, 2004)$. Desde mayo, Antonio Pérez Henares y Roberto Arce tomaron las riendas de La respuesta, pero pocas semanas después, a finales de junio, el programa desapareció de la parrilla de Antena 3, aunque muy pronto sería reemplazado por un espacio muy similar.

\section{Ruedo ibérico}

Ruedo ibérico fue un matinal presentado y dirigido por Montserrat Domínguez. El nuevo programa contaba con dos grandes bloques. En el primero, la presentadora y sus colaboradores realizaban una entrevista a un personaje relevante de la vida pública española. En el segundo, tenía lugar la tertulia política de temas de actualidad, "enfocada al análisis más que a la opinión" (Baragaño, 2004), en palabras de su directora.

Este espacio no solo ocupó el vacío que dejó La respuesta, sino que su duración se prolongó a dos horas en una primera etapa y se redujo paulatinamente debido a los bajos índices de audiencia (a partir de diciembre de 2004, 1 h 45 min; desde septiembre de 2005, 1 h 30 min). Para tratar de reflotar Ruedo ibérico, en 2006 se sumó al matinal Juanjo de la Iglesia al frente de la sección "El observatorio", donde realizaba "un análisis irreverente y lleno de ironía sobre las noticias, imágenes y titulares” (El Mundo, 2006) de la prensa escrita.

Sin embargo, este impulso no fue suficiente, y al igual que había sucedido con sus antecesores, el programa no tuvo la misma audiencia que sus principales competidores. Por ello, el 24 octubre de 2006, con un 10,4\% de audiencia $(A B C, 2006)$, finalizó la emisión de Ruedo ibérico que sería sustituido por Espejo público (Gallo, 2006). 


\section{Análisis cuantitativo: perfil de los entrevistados políticos en los matinales de TVE y Antena 3 (1997-2006)}

Tanto la cadena pública como la privada cedieron minutos de sus programas para entrevistar a personas destacadas, para completar el contenido de estos espacios centrados en debatir la actualidad política con tertulianos habituales procedentes principalmente del ámbito periodístico, como hemos visto. Entre 1997 y 2006, se han registrado 206 entrevistas distribuidas de manera muy equilibrada entre las dos cadenas objeto de estudio: 104 en el caso de TVE y 102 en Antena 3 (tabla 2).

\section{Tabla 2. Número de entrevistas: distribución por cadenas (1997-2006)}

\begin{tabular}{|c|c|c|}
\hline Año & TVE & Antena 3 \\
\hline 1997 & 12 & 10 \\
\hline 1998 & 10 & 10 \\
\hline 1999 & 11 & 11 \\
\hline 2000 & 8 & 9 \\
\hline 2001 & 10 & 9 \\
\hline 2002 & 14 & 10 \\
\hline 2003 & 9 & 8 \\
\hline 2004 & 9 & 13 \\
\hline 2005 & 12 & 11 \\
\hline 2006 & 9 & $\mathbf{1 0 2}$ \\
\hline Total & $\mathbf{1 0 4}$ & \\
\hline
\end{tabular}

Fuente: elaboración propia.

Cabe destacar, y de ahí el interés de este trabajo, que el porcentaje más elevado de entrevistas de los matinales se realizan a invitados que tenían relación con la política: o bien eran personas en activo, o bien no lo eran ya, pero estaban vinculadas abiertamente a una formación concreta. De las 206 entrevistas totales que se registraron, 155 invitados tenían este perfil, lo 
que supone contar con una muestra del $75,24 \%$ del total. Este porcentaje no varía notoriamente cuando se hace el estudio por cadenas: un 73,07 \% en la televisión pública y un 77,45 \% en la privada (figura 1).

\section{Figura 1. Número de entrevistas a invitados con perfil político: distribución por cadenas}

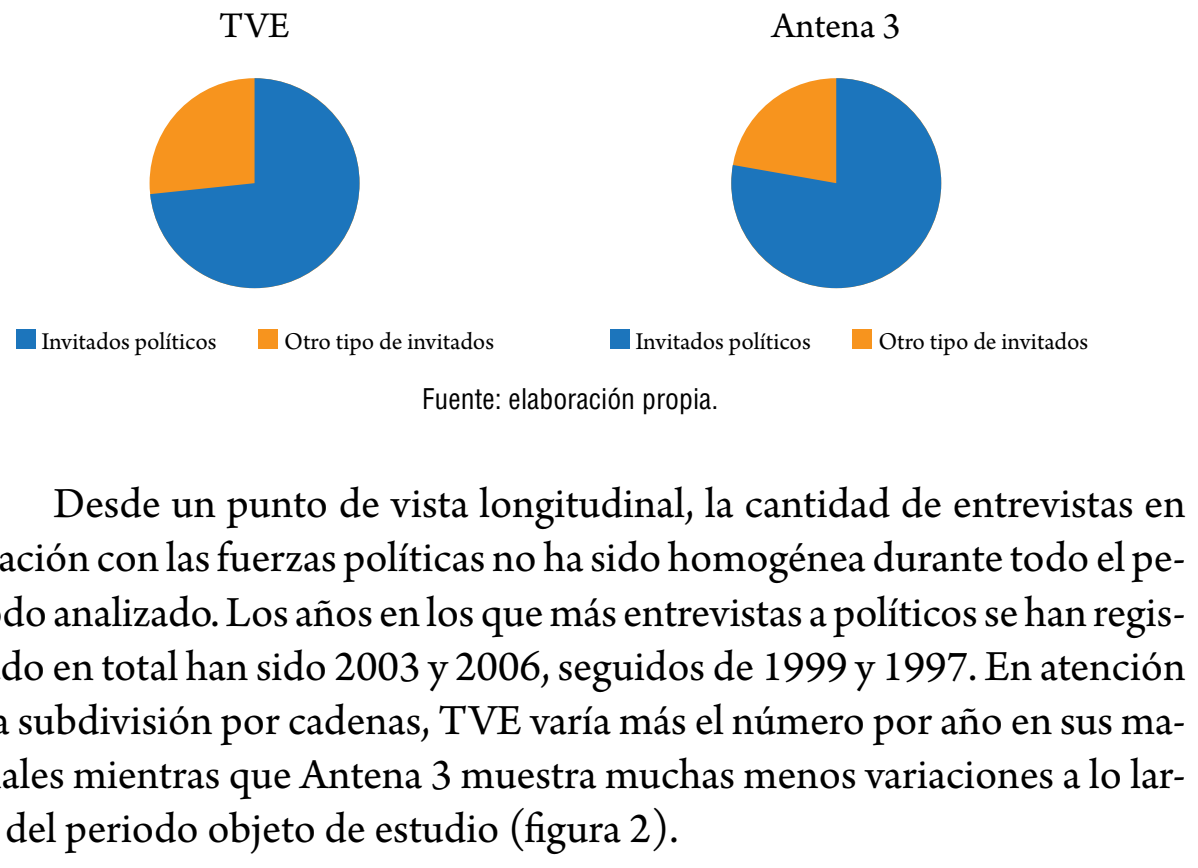

\section{Figura 2. Número de entrevistas a políticos por año: distribución por cadenas}

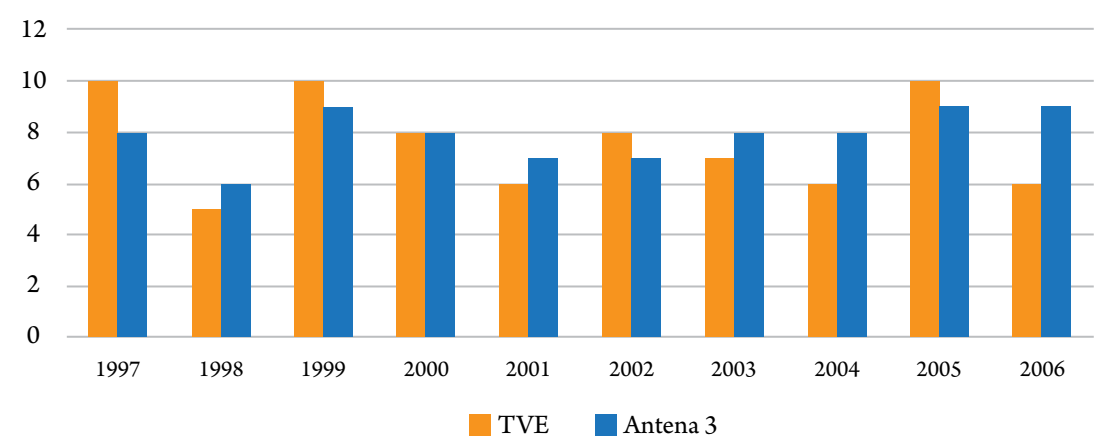

Fuente: elaboración propia. 
Otra de las variables analizadas tiene que ver con el partido político cuya marca estaba detrás de cada uno de los invitados que acudían a los programas matinales para ser entrevistados. De las 155 entrevistas, 67 se realizaron a miembros del PP, 51 del PSOE , 14 de Izquierda Unida (IU), 13 del nacionalismo catalán (Convergència i Unió [CiU]), 7 del Partido Nacionalista Vasco (PNV) y 3 de otros partidos (una a Coalición Canaria, una al Bloque Nacionalista Galego [BNG] y una al Gibraltar Social Democrats $[\mathrm{GSD}]$ ) (figura 3).

\section{Figura 3. Partidos políticos de los entrevistados en los matinales: distribución total}

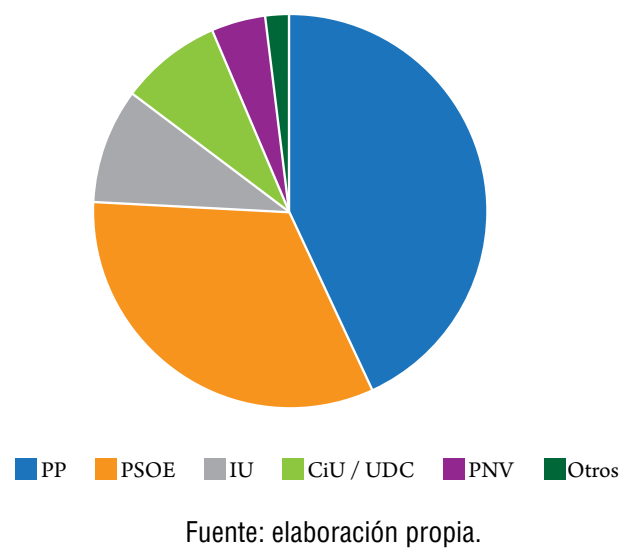

Si ajustamos el análisis haciendo un estudio por cadenas, las cifras no sufren variaciones importantes, aunque los matinales de Antena 3 realizaron un número ligeramente superior de entrevistas a políticos relacionados con el PP, el PSOE y PNV, mientras que TVE a miembros de IU y CiU/ Convergència Democràtica de Catalunya (CDC) (tabla 3).

\section{Tabla 3. Número de entrevistas por partido: distribución por cadenas}

\begin{tabular}{|c|c|c|}
\hline Partido político & TVE/La 2 & Antena 3 \\
\hline PP & 33 & 34 \\
\hline PSOE & 24 & 27 \\
\hline IU & 8 & 6 \\
\hline
\end{tabular}




\begin{tabular}{|c|c|c|}
\hline Partido político & TVE/La 2 & Antena 3 \\
\hline CiU/UDC & 8 & 5 \\
\hline PNV & 3 & 4 \\
\hline Otros & 0 & 3 \\
\hline Total & $\mathbf{7 6}$ & $\mathbf{7 9}$ \\
\hline
\end{tabular}

Fuente: elaboración propia.

Pese a la similitud de estas cifras, las apariciones de los políticos de un mismo partido tuvieron lugar en distintos momentos a lo largo del periodo analizado. Así, las entrevistas a miembros del PP han aparecido con mayor asiduidad en TVE entre 1997 y 2002, mientras que en Antena 3 lo hacían entre 1999 y 2003. En el caso de los invitados del PSOE, han aparecido con más afluencia en TVE entre 1999 y 2005, y en Antena 3 en 1997, 2004 y 2005 (figuras 4 y 5).

Figura 4. Tendencia partidista de los invitados en las entrevistas de los matinales de TVE: distribución por año

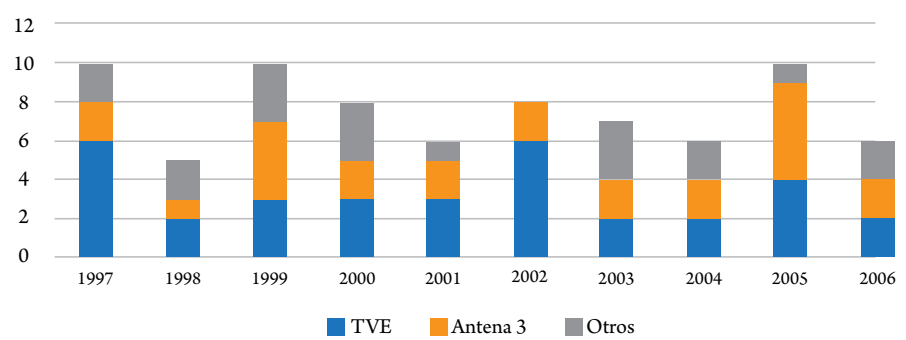

Fuente: elaboración propia.

Figura 5. Tendencia partidista de los invitados en las entrevistas de los matinales de Antena 3: distribución por año

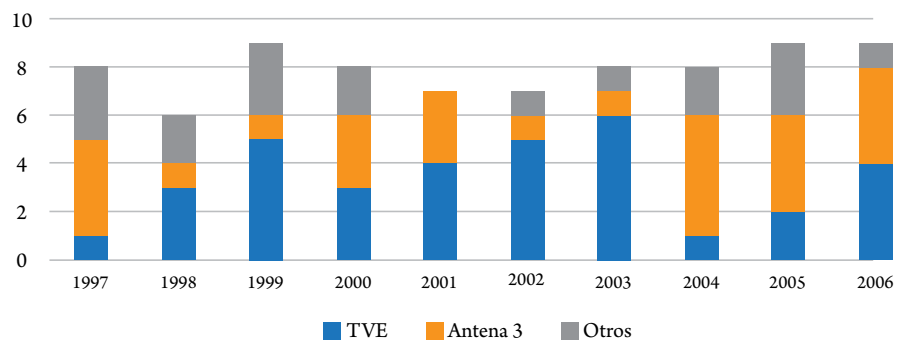

Fuente: elaboración propia. 
Sobre este punto, es destacable que ciertos políticos aparecieron varias veces en las entrevistas analizadas. Es el caso de Iñaki Anasagasti (tres apariciones en TVE y cuatro en Antena 3), José Antonio Duran i Lleida (cinco apariciones en TVE y dos en Antena 3) y Josep Piqué (cuatro apariciones en TVE y dos en Antena 3), entre otros (anexo 2). En el caso de las mujeres, son cinco las que aparecen en dos entrevistas: Celia Villalobos, Esperanza Aguirre, Loyola de Palacio, Ana Palacio y Rosa Díez. Por último, cabe señalar - llevando a cabo un análisis desde la perspectiva de género- que de las 155 entrevistas analizadas solo 25 se realizaron a mujeres políticas ( 15 en Antena 3 y 10 en TVE), lo cual refleja una presencia muy reducida frente a sus compañeros varones (figura 6).

\section{Figura 6. Entrevistas realizadas a políticos en los matinales de TVE y Antena 3: distribución por sexo}

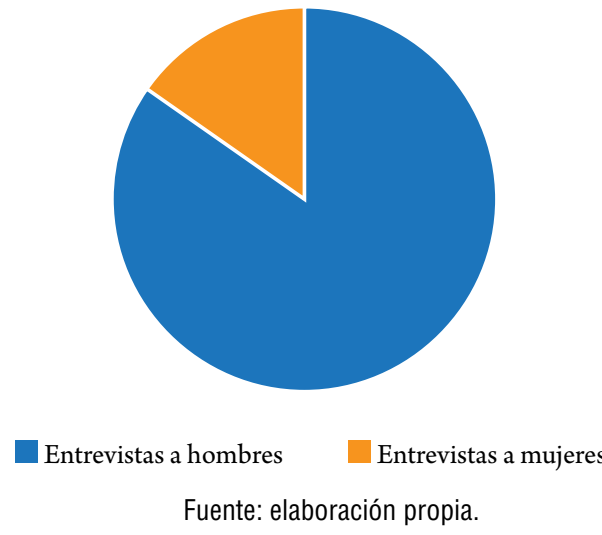

\section{Conclusiones}

Los datos extraídos de la muestra revelan que, pese a la dispar naturaleza de las dos cadenas analizadas - una pública y otra privada - ylos cambios de Gobierno acontecidos durante el periodo de investigación, se ha percibido en todo el estudio una variedad ideológica en los políticos invitados a la sección de entrevistas de los matinales de Antena 3 y TVE durante la primera década de su existencia. El mayor número de entrevistas realizadas corresponde a personas vinculadas a los dos principales partidos que han protagonizado el bipartidismo en el periodo histórico que corresponde con la etapa televisiva analizada. 
Así se entrevistaron mayoritariamente a miembros del PP (67) y del PSOE (51). Cabe destacar que no hay apenas diferencia entre el porcentaje de estas que llevó a cabo un canal u otro: en el caso del PP, un 49,25\% corresponden a TVE y un 50,75\% a Antena 3. En el del PSOE, un 47,06\% pertenecen a la cadena pública y un $52,94 \%$ a la privada.

También resulta llamativo que son políticos de los partidos nacionalistas en Cataluña y País Vasco (José Antonio Duran i Lleida e Iñaki Anasagasti) los que más veces aparecieron en los matinales: siete veces cada uno de ellos. Este hecho también guarda relación directa con el peso que en la política nacional tienen estos partidos, sobrerrepresentados en los programas matinales cuyas agendas se centran fundamentalmente en contenidos de actualidad política.

La aparición de los políticos en los programas matinales se produjo de manera constante entre 1997 y 2006. No obstante, es destacable que en estos espacios las mujeres tienen un peso muy dispar en relación con los hombres, y esta desigualdad se percibe de manera general tanto en la cadena pública como en la privada. Del total de entrevistas a políticos que se registran, solo el 16,13\% fueron a mujeres.

En definitiva, el análisis conjunto del estudio cualitativo y cuantitativo revela que los matinales que se emitieron en TVE y en Antena 3 fueron espacios muy similares, tanto en su estructura y su funcionamiento interno (periodistas y colaboradores) como en la relación convulsa entre la dirección de las cadenas y las de los programas o en el perfil de los invitados relacionados con la política que fueron entrevistados.

\section{Referencias}

ABC. (1994, enero 10). "Los desayunos de Radio 1" televisados en directo desde hoy, p. 125. Recuperado de http://hemeroteca.abc.es/nav/ Navigate.exe/hemeroteca/madrid/abc/1994/01/10/125.html

$A B C$. (2002, noviembre 27). Antena 3 destituye a Isabel San Sebastián de "El primer café". Recuperado de https://www.abc. 
es/espana/abci-antena-destituye-isabel-sebastian-primercafe-200211260300-146239_noticia.html

$A B C$. (2003, agosto 29). 'El primer café', de Antena 3, pasa a llamarse 'La respuesta', p. 92.

$A B C$. (2004, mayo 3). Pedro Piqueras asume la dirección de Radio Nacional de España. Recuperado de https://www.abc.es/ espana/abci-pedro-piqueras-asume-direccion-radio-nacional-espana-200405030300-9621298650592_noticia.html

ABC. (2006, octubre 27). La despedida de Montserrat Domínguez, p. 93.

$A B C$. (2012, agosto 21). María Casado releva a Ana Pastor en 'Los desayunos.'Recuperado de https://www.abc.es/tecnologia/rc-maria-casado-releva-pastor-201208200000_noticia.html

Baragaño, T. (2004, septiembre 4). Montserrat Domínguez dirige desde el lunes cinco horas de informativo matinal en Antena 3. El País. Recuperado https://elpais.com/diario/2004/09/04/radiotv/1094248801_850215.html

Diario de sesiones del Congreso de los Diputados, 25 de septiembre de 1996.

Diario de sesiones del Congreso de los Diputados, 3 de junio de 1997.

Diario de sesiones del Congreso de los Diputados, 29 de octubre de 1998.

Diario de sesiones del Congreso de los Diputados, 21 de febrero de 2001.

El Confidencial. (2016, julio 28). Sergio Martín presentará 'Los desayunos de TVE' en sustitución de María Casado. Recuperado de https:// www.elconfidencialdigital.com/articulo/medios/Sergio-MartinTVE-Maria-Casado/20160727182842082259.html 
El Mundo. (2004, febrero 20). Pedro Piqueras se hará cargo del espacio matinal de Antena 3 'La respuesta', que conducía Ferrari. Recuperado de https://www.elmundo.es/elmundo/2004/02/20/comunicacion/1077282495.html

El Mundo. (2006, abril 28). Juanjo de la Iglesia se incorpora a 'Ruedo ibérico' para hacer un resumen de prensa diario. Recuperado de https://www.elmundo.es/elmundo/2006/04/27/comunicacion/1146150000.html

El País. (1997, enero 22). Álvarez de Toledo dimite de 'El primer café. https:// elpais.com/diario/1997/01/22/radiotv/853887602_850215.html

El País. (1998, junio 26). Isabel San Sebastián deja TVE y ficha por Antena 3. Recuperado de https:/ / elpais.com/diario/1998/06/26/radiotv/898812009_850215.html

El País. (2004, julio 13). Pepa Bueno dirigirá la tertulia de TVE 'Los desayunos'. Recuperado de https://elpais.com/diario/2004/07/13/ radiotv/1089669601_850215.html

El País. (2012, agosto 20). María Casado sustituirá a Ana Pastor al frente de 'Los desayunos de TVE'. Recuperado de https://elpais.com/cultura/2012/08/20/television/1345470890_536125.html

Gallo, I. (2003a, agosto 25). 'El primer café', de Antena 3, pasa a llamarse 'La respuesta'. El País.

Gallo, I. (2003b, agosto 29). Ferrari recupera a Carlos Herrera para la tertulia matinal de Antena 3. El País. Recuperado de https://elpais. com/diario/2003/08/29/radiotv/1062108002_850215.html

Gallo, I. (2006, noviembre 23). Antena 3 cierra mañana la tertulia política 'Ruedo ibérico'. El País. Recuperado de https://elpais.com/diario/2006/11/23/radiotv/1164236403_850215.html 
García Mirón, S. (2014a). Acercamiento al estilo de Antena 3 en la Etapa Asensio (1992-1997): evolución empresarial, contenidos, identidad y audiencia. Sphera Pública, 2(14), 80-103. Recuperado de http://sphera.ucam.edu/index.php/sphera-01/article/view/230

García Mirón, S. (2014b). Antena 3, nacimiento y evolución (1990-2010): contenidos, estilo y estrategias de programación en prime time de la primera emisora de televisión privada española (Tesis de doctorado, Universida de Vigo, Vigo, España). Recuperado de http://www. investigo.biblioteca.uvigo.es/xmlui/handle/11093/143

García Mirón, S. (2015). La creación de estrategias de programación a partir de la sinergia entre medios: el caso de Antena 3. En El nuevo diálogo social organizaciones, públicos y ciudadanos. (pp. 365-382). Valencia, España: Campgráfic.

Gómez-Escalonilla, G. (2003). Programar televisión: análisis de los primeros cuarenta años de programación televisiva en España. Madrid, España: Dykinson.

Gómez Montano, A. (2006). La manipulación en televisión. Madrid, España: Espejo de Tinta.

Gómez, R. (2012, agosto 4). Ana Pastor: "Me destituyen por hacer periodismo". El País. Recuperado de https://elpais.com/socie$\mathrm{dad} / 2012 / 08 / 04 /$ actualidad/1344081821_727397.html

Humanes, M. L. y Fernández Alonso, M. I. (2015). Pluralismo informativo y medios públicos: la involución de TVE en el contexto del cambio político (2012-2013). Revista Latina de Comunicación Social, 70, 270-287. https://doi.org/10.4185/RLCS-2015-1046

La Vanguardia. (2002, noviembre 26). Antena 3 destituye a Isabel San Sebastián y pone a Carmen Gurruchaga al frente de 'El primer café. Recuperado de https://www.lavanguardia.com/ 
vida/20021126/51262765270/antena-3-destituye-a-isabel-sansebastian-y-pone-a-carmen-gurruchaga-al-frente-de-el-primercafe.html

Martín Jiménez, V. (2018). Programación y estrategias de programación en la Transición. En J. Montero Díaz (Coord.), Una televisión con dos cadenas: la programación en España (1956-1990). (pp. 319-334). Madrid, España: Cátedra.

Montero Díaz, J. y Antona Jimeno, T. (2018). Programación y estrategias de programación en la televisión franquista. En J. Montero Díaz (Coord.), Una televisión con dos cadenas: la programación en España (1956-1990). (pp. 21-38). Madrid, España: Cátedra.

Palacio, M. (2007). La televisión pública española (TVE) en la era de José Luis Rodríguez Zapatero. Journal of Spanish Cultural Studies, 8(1), 71-83. https://doi.org/10.1080/14636200601148843

Radiotelevisión Española. (s. f.). Directores generales de Radiotelevisión Española. Recuperado de http://www.rtve.es/fotogalerias/directores-generales-radiotelevision-espanola/195335/

TVE. (2018). Más desayunos. Recuperado de http://www.rtve.es/alacarta/videos/mas-desayunos/

Vinuesa Tejero, M. L., Abejón Mendoza, P.y Sánchez Calero, M. L. (2011). Mujeres y política: un binomio con baja representación. Ámbitos: Revista Internacional de Comunicación, 20, 127-145. Recuperado de https://idus.us.es/xmlui/handle/11441/67584 


\section{Anexos}

\section{Anexo 1: Programas analizados: distribución por cadenas y meses}

\begin{tabular}{|c|c|c|}
\hline Semanas de estudio & TVE/La 2 & Antena 3 \\
\hline 10-16 de marzo de 1997 & 5 & 5 \\
\hline 14-20 de julio de 1997 & 5 & 0 \\
\hline 22-28 de septiembre de 1997 & 5 & 5 \\
\hline 22-28 de diciembre de 1997 & 0 & 0 \\
\hline 9-15 de marzo de 1998 & 5 & 5 \\
\hline 13-19 de julio de 1998 & 0 & 0 \\
\hline 21-27 de septiembre de 1998 & 5 & 5 \\
\hline 21-27 de diciembre de 1998 & 0 & 1 \\
\hline 15-21 de marzo de 1999 & 4 & 4 \\
\hline 12-18 de julio de 1999 & 0 & 0 \\
\hline 20-26 de septiembre de 1999 & 5 & 5 \\
\hline 20-26 de diciembre de 1999 & 2 & 2 \\
\hline 13-19 de marzo de 2000 & 5 & 5 \\
\hline $10-16$ de julio de 2000 & 0 & 0 \\
\hline 18-24 de septiembre de 2000 & 5 & 5 \\
\hline 25-31 de diciembre de 2000 & 0 & 0 \\
\hline 12-18 de marzo de 2001 & 5 & 5 \\
\hline 9-15 de julio de 2001 & 0 & 0 \\
\hline 24-29 de septiembre de 2001 & 5 & 5 \\
\hline 24-30 de diciembre de 2001 & 0 & 0 \\
\hline 13-17 de marzo de 2002 & 5 & 5 \\
\hline 8-14 de julio de 2002 & 5 & 0 \\
\hline $23-29$ de septiembre de 2002 & 5 & 5 \\
\hline 23-29 de diciembre de 2002 & 2 & 0 \\
\hline 10-16 de marzo de 2003 & 5 & 5 \\
\hline 14-20 de julio de 2003 & 0 & 0 \\
\hline
\end{tabular}




\begin{tabular}{|c|c|c|}
\hline Semanas de estudio & TVE/La 2 & Antena 3 \\
\hline 22-28 de septiembre de 2003 & 5 & 4 \\
\hline 22-28 de diciembre de 2003 & 0 & 0 \\
\hline 15-21 de marzo de 2004 & 5 & 5 \\
\hline 12-18 de julio de 2004 & 0 & 0 \\
\hline 20-26 de septiembre de 2004 & 5 & 5 \\
\hline 20-26 de diciembre de 2004 & 2 & 3 \\
\hline 14-20 de marzo de 2005 & 5 & 5 \\
\hline 11-17 de julio de 2005 & 0 & 0 \\
\hline 19-25 de septiembre de 2005 & 5 & 5 \\
\hline $19-25$ de diciembre de 2005 & 3 & 3 \\
\hline 13-19 de marzo de 2006 & 5 & 5 \\
\hline $10-16$ de julio de 2006 & 0 & 0 \\
\hline 18-29 de septiembre de 2006 & 5 & 5 \\
\hline 25-31 de diciembre de 2006 & 0 & 0 \\
\hline Total & 118 & 107 \\
\hline
\end{tabular}

Fuente: elaboración propia. 


\section{Anexo 2: Número de apariciones de cada entrevistado político (1997-2006)}

\begin{tabular}{|c|c|c|c|}
\hline Invitado & Partido & N.o apariciones & Año y canal de emisión \\
\hline Iñaki Anasagasti & PNV & 7 & $\begin{array}{c}\text { 1997-Antena } 3 \\
\text { 1998-TVE } \\
\text { 1998-Antena } 3 \\
\text { 1999-Antena } 3(2) \\
\text { 2001-TVE } \\
\text { 2003-TVE }\end{array}$ \\
\hline José Antonio Duran i Lleida & UDC & 7 & $\begin{array}{l}\text { 1998-TVE } \\
\text { 1999-TVE } \\
\text { 2000-TVE } \\
\text { 2000-Antena } 3 \\
\text { 2004-Antena } 3 \\
\text { 2005-TVE } \\
\text { 2006-TVE }\end{array}$ \\
\hline Josep Piqué & $\mathrm{PP}$ & 6 & $\begin{array}{c}\text { 1998-TVE } \\
\text { 2000-Antena } 3 \\
\text { 2002-TVE } \\
\text { 2004-TVE } \\
\text { 2006-TVE } \\
\text { 2006-Antena } 3\end{array}$ \\
\hline Rodrigo Rato & $\mathrm{PP}$ & 5 & $\begin{array}{c}\text { 1998-Antena } 3 \\
\text { 1999-TVE } \\
\text { 2000-TVE } \\
\text { 2001-Antena } 3 \\
\text { 2002-Antena } 3\end{array}$ \\
\hline Jesús Caldera & PSOE & 5 & $\begin{array}{l}\text { 2000-Antena } 3 \\
\text { 2001-Antena } 3 \\
\text { 2004-TVE } \\
\text { 2005-Antena } 3 \\
\text { 2006-TVE }\end{array}$ \\
\hline José Bono & PSOE & 5 & $\begin{array}{c}\text { 1997-Antena } 3 \\
\text { 2000-Antena } 3 \\
\text { 2002-TVE } \\
\text { 2005-TVE } \\
\text { 2006-Antena } 3\end{array}$ \\
\hline Jaime Mayor Oreja & PP & 4 & $\begin{array}{l}\text { 1998-Antena } 3 \\
\text { 1999-Antena } 3 \\
\text { 2000-TVE } \\
\text { 2005-Antena } 3\end{array}$ \\
\hline Juan Fernando López Aguilar & PSOE & 4 & $\begin{array}{c}\text { 2001-Antena } 3 \\
\text { 2003-TVE } \\
\text { 2004-TVE }\end{array}$ \\
\hline
\end{tabular}




\begin{tabular}{|c|c|c|c|}
\hline Invitado & Partido & N. ${ }^{\circ}$ apariciones & Año y canal de emisión \\
\hline Julio Anguita & IU & 4 & $\begin{array}{c}\text { 1997-TVE } \\
\text { 2000-TVE } \\
\text { 2001-Antena } 3 \\
\text { 2006-TVE }\end{array}$ \\
\hline Federico Trillo & PP & 3 & $\begin{array}{c}\text { 2001-TVE } \\
\text { 2005-Antena } 3 \\
\text { 2006-Antena } 3\end{array}$ \\
\hline Carlos Iturgaiz & PP & 3 & $\begin{array}{c}\text { 1997-TVE } \\
\text { 2000-Antena } 3 \\
\text { 2003-TVE }\end{array}$ \\
\hline Javier Arenas & $\mathrm{PP}$ & 3 & $\begin{array}{c}\text { 2000-Antena } 3 \\
\text { 2003-TVE } \\
\text { 2003-Antena } 3\end{array}$ \\
\hline Alfredo Pérez Rubalcaba & PSOE & 3 & $\begin{array}{c}\text { 1998-TVE } \\
\text { 1999-TVE } \\
\text { 2004-Antena } 3\end{array}$ \\
\hline Manuel Chaves & PSOE & 3 & $\begin{array}{l}\text { 1999-TVE } \\
\text { 2005-TVE } \\
\text { 2006-TVE }\end{array}$ \\
\hline Francisco Frutos & IU & 3 & $\begin{array}{c}\text { 1999-TVE } \\
\text { 2000-TVE } \\
\text { 2000-Antena } 3\end{array}$ \\
\hline Gaspar Llamazares & IU & 3 & $\begin{array}{c}\text { 2003-TVE } \\
\text { 2004-TVE } \\
\text { 2004-Antena } 3\end{array}$ \\
\hline Celia Villalobos & $\mathrm{PP}$ & 2 & $\begin{array}{c}\text { 2001-Antena } 3 \\
\text { 2002-TVE }\end{array}$ \\
\hline Manuel Fraga & $\mathrm{PP}$ & 2 & $\begin{array}{c}\text { 1999-TVE } \\
\text { 2001-Antena } 3\end{array}$ \\
\hline Esperanza Aguirre & PP & 2 & $\begin{array}{c}\text { 1999-TVE } \\
\text { 2001-Antena } 3\end{array}$ \\
\hline Loyola de Palacio & $\mathrm{PP}$ & 2 & $\begin{array}{l}\text { 1998-Antena } 3 \\
\text { 1999-Antena } 3\end{array}$ \\
\hline Ana Palacio & $\mathrm{PP}$ & 2 & $\begin{array}{l}\text { 2002-Antena } 3 \\
\text { 2003-Antena } 3\end{array}$ \\
\hline Luis de Grandes & $\mathrm{PP}$ & 2 & $\begin{array}{l}\text { 1997-TVE } \\
\text { 2002-TVE }\end{array}$ \\
\hline Ángel Acebes & PP & 2 & $\begin{array}{l}\text { 1997-Antena } 3 \\
\text { 2005-Antena } 3\end{array}$ \\
\hline Alberto Ruiz Gallardón & PP & 2 & $\begin{array}{l}\text { 2002-Antena } 3 \\
\text { 2003-Antena } 3\end{array}$ \\
\hline
\end{tabular}




\begin{tabular}{|c|c|c|c|}
\hline Invitado & Partido & N.o apariciones & Año y canal de emisión \\
\hline Mariano Rajoy & $\mathrm{PP}$ & 2 & $\begin{array}{l}\text { 2002-Antena } 3 \\
\text { 2003-Antena } 3\end{array}$ \\
\hline Gabriel Elorriaga & $\mathrm{PP}$ & 2 & $\begin{array}{l}\text { 2004-TVE } \\
\text { 2005-TVE }\end{array}$ \\
\hline Francisco Camps & $\mathrm{PP}$ & 2 & $\begin{array}{l}\text { 2004-Antena } 3 \\
\text { 2005-Antena } 3\end{array}$ \\
\hline Pedro Solbes & PSOE & 2 & $\begin{array}{l}\text { 1999-TVE } \\
\text { 2005-TVE }\end{array}$ \\
\hline Carlos Solchaga & PSOE & 2 & $\begin{array}{c}\text { 1997-TVE } \\
\text { 1997-Antena } 3\end{array}$ \\
\hline Nicolás Redondo & PSOE & 2 & $\begin{array}{l}\text { 1997-TVE } \\
\text { 2000-TVE }\end{array}$ \\
\hline Enrique Múgica & PSOE & 2 & $\begin{array}{c}\text { 1997-Antena } 3 \\
\text { 2000-TVE }\end{array}$ \\
\hline Juan Carlos Rodríguez Ibarra & PSOE & 2 & $\begin{array}{l}\text { 1999-Antena } 3 \\
\text { 2000-Antena } 3\end{array}$ \\
\hline Rosa Díez & PSOE & 2 & $\begin{array}{l}\text { 2001-Antena } 3 \\
\text { 2005-Antena } 3\end{array}$ \\
\hline Artur Mas & $\mathrm{CiU}$ & 2 & $\begin{array}{c}\text { 2002-Antena } 3 \\
\text { 2004-TVE }\end{array}$ \\
\hline Juan Ignacio Barrero & PP & 1 & 1997-TVE \\
\hline Juan Van Halen & PP & 1 & 1997-TVE \\
\hline Amalia Gómez & PP & 1 & 1998-TVE \\
\hline Sergio Marqués & $\mathrm{PP}$ & 1 & 1998-Antena 3 \\
\hline Isabel Tocino & PP & 1 & 1999-TVE \\
\hline Aleix Vidal Quadras & $\mathrm{PP}$ & 1 & 1999-Antena 3 \\
\hline Manuel Pimentel & $\mathrm{PP}$ & 1 & 1999-Antena 3 \\
\hline Alberto Fernández Díaz & PP & 1 & 1999-Antena 3 \\
\hline José María Michavila & $\mathrm{PP}$ & 1 & 1999-Antena 3 \\
\hline Pío Cabanillas & $\mathrm{PP}$ & 1 & 2000-TVE \\
\hline Juan Carlos Aparicio & $\mathrm{PP}$ & 1 & 2001-TVE \\
\hline Rafael Bardají & $\mathrm{PP}$ & 1 & 2001-TVE \\
\hline Gonzalo Robles & PP & 1 & 2002-TVE \\
\hline Juan Costa & PP & 1 & 2002-TVE \\
\hline Ana Pastor & $\mathrm{PP}$ & 1 & 2002-TVE \\
\hline
\end{tabular}




\begin{tabular}{|c|c|c|c|}
\hline Invitado & Partido & N. ${ }^{\circ}$ apariciones & Año y canal de emisión \\
\hline Pilar del Castillo & PP & 1 & 2002-Antena 3 \\
\hline Eduardo Zaplana & $\mathrm{PP}$ & 1 & 2003-Antena 3 \\
\hline Elvira Rodríguez & PP & 1 & 2003-Antena 3 \\
\hline Ramón Luis Valcárcel & PP & 1 & 2005-TVE \\
\hline María San Gil & $\mathrm{PP}$ & 1 & 2005-TVE \\
\hline Ignacio Uriarte & $\mathrm{PP}$ & 1 & 2006-Antena 3 \\
\hline Juan Alberto Belloch & PSOE & 1 & 1997-Antena 3 \\
\hline Carlos Totorica & PSOE & 1 & 1998-Antena 3 \\
\hline Ángeles Amador & PSOE & 1 & 1999-TVE \\
\hline Consuelo Rumi & PSOE & 1 & 2001-TVE \\
\hline Micaela Navarro & PSOE & 1 & 2001-TVE \\
\hline Javier Solana & PSOE & 1 & 2002-TVE \\
\hline Javier Rojo & PSOE & 1 & 2002-Antena 3 \\
\hline Emilio Menéndez del Valle & PSOE & 1 & 2003-TVE \\
\hline Manuel Marín & PSOE & 1 & 2003-Antena 3 \\
\hline Alfonso Guerra & PSOE & 1 & 2004-Antena 3 \\
\hline Cristina Alberdi & PSOE & 1 & 2004-Antena 3 \\
\hline Elena Salgado & PSOE & 1 & 2004-Antena 3 \\
\hline Teresa Fernández de la Vega & PSOE & 1 & 2004-Antena 3 \\
\hline Odon Elorza & PSOE & 1 & 2005-TVE \\
\hline Joan Clos & PSOE & 1 & 2005-TVE \\
\hline Carmen Calvo & PSOE & 1 & 2005-Antena 3 \\
\hline Fernando Moraleda & PSOE & 1 & 2005-Antena 3 \\
\hline José Blanco & PSOE & 1 & 2006-Antena 3 \\
\hline José Montilla & PSOE & 1 & 2006-Antena 3 \\
\hline Antonio Romero & $\mathrm{IU}$ & 1 & 1997-TVE \\
\hline Cristina Almeida & $\mathrm{IU}$ & 1 & 1997-Antena 3 \\
\hline Felipe Alcaraz & $\mathrm{IU}$ & 1 & 1999-TVE \\
\hline Rosa Aguilar & $\mathrm{IU}$ & 1 & 2006-TVE \\
\hline Pere Esteve & $\mathrm{CiU}$ & 1 & 1997-TVE \\
\hline Joaquín Molins & $\mathrm{CiU}$ & 1 & 1997-Antena 3 \\
\hline
\end{tabular}




\begin{tabular}{|c|c|c|c|}
\hline Invitado & Partido & N. ${ }^{\mathbf{a}}$ apariciones & Año y canal de emisión \\
\hline Ignasi Guardans & $\mathrm{CiU}$ & 1 & 2003-TVE \\
\hline Jordi Pujol & CiU & 1 & 2005-Antena 3 \\
\hline José Carlos Mauricio & CC & 1 & 2003-Antena 3 \\
\hline Anxo Quintana & BNG & 1 & 2005-Antena 3 \\
\hline Peter Caruana & GSD & 1 & 2006-TVE \\
\hline
\end{tabular}

Fuente: elaboración propia. 